\title{
Widening the Field and Sparks of the Future
}

\author{
Negin Mirriahi, Shane Dawson, \\ Dragan Gasevic, and Phil Long
}

Editors, Journal of Learning Analytics

\begin{abstract}
This issue of the Journal of Learning Analytics comprises two special issue sections. The first of which presents five papers from the 4th International Learning Analytics and Knowledge conference held in Indianapolis. The second showcases the current or recent work of doctoral students who attended the 2nd Learning Analytics Summer Institute at Harvard University, Boston. The issue also includes two articles in the Hot Spots section, discussing the application of learning analytics initiatives in higher education institutions from different perspectives - broad-scale initiatives to individual course design. The breadth and diversity of the articles covered in this issue demonstrate how the discipline has matured and moved towards understanding student learning to inform pedagogical practice and curricular redesign coupled with strategies for the application and adoption of LA strategies across institutions
\end{abstract}

KEYWORDS: Special issue, learning analytics, research, practice, Society for Learning Analytics Research, SoLAR, LAK'14, LASI'14

\section{Editorial}

Three separate yet complementary sections comprise this concluding issue of the first volume of the Journal of Learning Analytics (JLA). As the journal concludes its first year of publishing, the papers presented in this issue demonstrate how the learning analytics (LA) research has grown and diversified alongside the substantial advancements in the application of LA initiatives within higher education institutions. As the field was developing the LA research largely focused on how analytics can help predict attrition and academic performance to inform institutional strategies for improving student retention. As evident in this latest issue, many researchers and practitioners are now focusing their efforts on using LA methodologies, often through inter-disciplinary teams, for better understanding how students learn in order to guide and inform pedagogical practice, course design, curriculum, learning support, and the development of effective learning communities to fundamentally improve their academic experience. While research on student learning is not novel nor a unique discipline, the rapid adoption of online technologies by academic staff either by their own volition or due to blended, online, flexible, and personalised learning strategies that are being encouraged in many higher education institutions has given rise to massive datasets that allows for more sophisticated analysis of a variety of objective data (and in many cases, triangulated with self-informed data as well) providing insight into how students actually experience learning. As the availability of data on students' engagement with online technologies continues to grow and LA researchers continue to push the box in terms of the 
application of methodologies previously not applied to research on student learning, our collective understanding of how, when, why, and with whom students learn will only flourish.

A part of the rapid evolution of the discipline can be attributed to the growth in the number of established researchers coming together from various disciplines (e.g. education, computer science, statistics, etc.) to investigate the breadth of learning and teaching related data. However the future expansion and research directions will be in the hands of a growing number of post-graduate and doctoral students. While the LA field continues to better understand the student learning process and raise awareness of learning and teaching issues, its capacity for future research is fostered through the development of such postgraduate and doctoral students who not only develop new and innovative methodologies and uncover answers to challenging questions, but their research motivates and attracts the investigation of further research questions. Hence, developing the research capacity and interest in the field will further fuel the longer term development, of the discipline.

Finally, critical to the discipline and for sustaining its impact on the education sector (K-12 or higher education), is the articulation of LA research into day to day practice. As well noted in this issue, whether it is to inform course redesign, curriculum reform, student support strategies, or early alert dashboards or intervention mechanisms, as research in the discipline diversifies and matures, its application in real-world settings through institutional or faculty adoption, is paramount. While, the application of LA is often fraught with challenges, institutions are beginning to identify solutions that can lead to wide-scale application. However, as a LA community, we need to share how institutional challenges can be overcome in order to advance LA practice alongside the continued surge and progression of new discoveries through research.

\subsection{Selected and Invited Papers Presented at LAK'14}

This issue begins with a special issue led by the guest editors, Abelardo Pardo and Stephanie Teasley, who have compiled a selection of representative papers from the 4th International Learning Analytics and Knowledge Conference (LAK'14). The research presented in the five papers demonstrates the diversification of LA research and the move towards greater investigation into student learning and making sense of the 'data exhaust' for actionable insight that can transfer to practice impacting students, instructors, programs, and institutions (Pardo \& Teasley, 2014). The papers cover various themes such as discourse analysis of discussion forums, students' use and engagement with e-portfolios and course resources, curriculum issues, and large-scale adoption of LA strategies. Collectively, they showcase the current breadth of LA research and how it can inform pedagogical practice, course and curriculum redesign, and institutional adoption. We thank Abelardo and Stephanie for their hard work and dedication for bringing this special issue to fruition. 
(2014). Widening the Field and Sparks of the Future, 1(3), 1-3.

\subsection{Sparks of the Learning Analytics Future}

A central aim established by the Socioety for Learning Analytics Research (SoLAR) relates to the building of research capacity in learning analytics. To achieve this aim, SoLAR has held numerous events specifically for Doctoral and Post-graduate students. The Learning Analytics Summer Institute (LASI) is one such. The second segment of this issue, led by Dragan Gasevic and Mykola Pechenizkiy, presents the current or recent work of doctoral students conducting their research in the LA discipline who attended the 2nd Learning Analytics Summer Institute (LASI'14). While the students had an opportunity to discuss their research with each other and LA researchers through lightening talks and poster presentations at LASI'14, they were invited to submit a brief article to this issue sharing their research with the wider community. As the sixteen articles in this section demonstrate, doctoral research in LA has built momentum and the diversity of research questions, datasets, and novel methodologies continues to grow. This section provides the unique opportunity to glimpse into the future of LA research as reveals what the community can expect to see and learn in the near future from these rising researchers. We thank Dragan and Mykola for compiling this special issue and encouraging the doctoral students for sharing their work with the JLA readership.

\subsection{Hot Spots}

The third and final segment of this issue includes two papers that discuss the application of LA research to practice. With one paper focusing on the challenges institutions face with establishing data governance models for LA initiatives and the other reporting on a tool that marries analytics with design to help teaching staff readily access the relevant data to inform their course design decisions, these papers illustrate the growing adoption of LA practice in higher education institutions and the strategies that can best support it. The first paper, Critical Factors in Data Governance for LA by Elouazizi, discusses the challenges facing institutions who are grappling with developing data governance models for LA initiatives. Overlaps between the various types of LA data, data sources, and stakeholder groups are explored and three main challenges are identified with seven key suggestions for how institutions can overcome them. The second paper by Dunbar, Dingel, Prat-Resina, reports on the Browser of Student and Course Objects (BoSCO), a LA tool that bridges the analytics and course design areas by providing teaching staff with access to data that is most relevant and useful for informing their course and curricular design decisions. 